legal and clinical implications. Further discussion needs to take place to seek an agreed way forward in this important yet difficult area.

\section{Reference}

BARNES, T. R. E., MCEVEDY, C. J. B. \& Nelson, H. E. (1996) Management of treatment-resistant schizophrenia unresponsive to clozapine. British Journal of Psychiatry, 169 (suppl. 31), 31-40.

BRTISH NATIONAL FORMULARY (1998) British National Formulary. Volume 36. London: British Medical Association and the Royal Pharmaceutical Society of Great Britain.

KANE, J. M. (1992) Clinical efficacy of clozapine in treatment-refractory schizophrenia: an overview. British Journal of Psychiatry, 160 (suppl.17), 41-45.
Mental Health ACt Commission (1993) Guidance on the administration of clozapine and other treatments requiring blood tests under the provision of Part IV of the Mental Health Act: Practice Note 1. Nottingham: Mental Health Act Commission.

MORTIMER, A. (1996) Using Clozarll under the Mental Health Act. Clozaril Patient Monitoring Service Newsletter. Issue 14. Camberley: Sandoz Pharmaceuticals.

*Stephen Pereira, Consultant Psychiatrist, Oxleas NHS Trust, 132/134 Powis Street, Woolwich, London SE18 6NL; Dominic Beer, Consultant Psychiatrist, and Carol Paton, Principal Pharmacist, Oxleas NHS Trust

*Correspondence

\title{
Commentary: The risks of enforcing clozapine therapy
}

\author{
Thomas R. E. Barnes
}

Pereira et als paper (1999, this issue) is to be welcomed in that it highlights an area of clinical decision that requires a careful balance of short- and long-term risks and benefits in the individual patient. In this (necessarily) brief commentary I will concentrate on this aspect, and leave aside any ethical and medico-legal considerations.

The essence of this paper is a "locally devised structured decision process" for enforcing clozapine therapy in patients for whom it is indicated, but who are unwilling to take it. The paper presents a structured decision 'aide memoire', which is rather non-specific. For example, what constitutes a lack of response to previous antipsychotic medication is not defined in terms of adequate dosage, duration or adherence. Further, the authors recommend the broad canvassing of general views from colleagues and official bodies. However, there is a distinction to be made here between an informed second opinion relating to a particular patient, hearing of other clinicians' experience of starting patients on clozapine and informal discussion about the suggested approach.

Perhaps most critically, there is no mention of the need to elicit exactly why an individual patient might be currently reluctant to start clozapine. Depending on the reasons, the patient may be amenable to change through strategies such as reassurance and more detailed information about the potential hazards and advantages of the drug, or a psychological intervention, specifically cognitive-behavioural therapy, to improve aspects of insight or tackle a particular delusion (Barnes et al, 1996). Discussion between the patient and others already receiving clozapine may serve to allay concerns. Using such an approach in our in-patient service for treatment-resistant schizophrenia, along with patience and steady persuasion, we have usually achieved the goal of the patient eventually accepting treatment. If not, the risks, both short-term and long-term, of confrontation have generally been judged to outweigh the potential benefits, and the plan to administer clozapine has been abandoned, or at least postponed.

The possible short-term benefits of enforcing clozapine in a particular patient, in the manner described by Pereira et al are that a blood sample is obtained and clozapine treatment initiated. The risks include needle-stick injury, disruption of therapeutic relationships and problems asso- 
ciated with subsequent non-adherence with the treatment regime. Further, such an approach may prove divisive for the clinical team, with members having different views about its advisability. Such uncertainty and ambivalence among team members is likely to have an adverse effect on the patient. In the longer term, the most positive outcome is that the patient shows persistent clinical improvement, and willingly adheres with both medication and the blood tests, as the reasons for the original reluctance to take clozapine no longer hold sway. In theory, there are several plausible explanations for such a change. For example, if the objections to clozapine or the blood tests were delusionally-based they might be overcome by a reduction in the influence and intensity of the delusions as part of the treatment response. If the original concern was about possible sideeffects, the patient may be reassured by the experience of taking the medication. However, in the absence of any published data on the clinical outcome following the initial enforcement of a clozapine regime, such long-term benefits remain theoretical. The potential for a positive outcome needs to be balanced against the possible long-term adverse consequences of enforcing clozapine. The drug may fail to achieve the degree of improvement (in terms of reduction in the intensity of relevant delusional ideas, improvement in insight, etc.) that would render the patient likely to comply with medication over time. If therapeutic relationships with the multidisciplinary team are not re-established, the patient may disengage from treatment and any broader rehabilitation programme. Even if this does not occur, discussions between members of the clinical team and the patient may become limited to an unproductive debate about adherence to the medication regime, at the expense of consideration of wider issues related to rehabilitation and social integration.

While the potential risks and benefits of enforcing clozapine are uncertain in those patients who have never been exposed to the drug, more reliable predictions may be made for those who have already received an adequate trial. For patients previously showing an impressive improvement with clozapine, who are otherwise unresponsive to antipsychotic medication, it could be argued that the justification is rather greater (Barnes et al, 1996). This may be particularly so for patients who pose a 'substantial risk of harm to others or themselves' related to the severity of their psychotic illness. In such cases, the successful use of electroconvulsive therapy to gain a temporary improvement in the psychotic illness, allowing cooperation with the clozapine regime, has been reported (Green et al, 1994; James \& Gray, 1999).

\section{References}

BARNES, T. R. E., MCEvedy, C. J. B. \& NELSON, H. E. (1996) Management of treatment resistant schizophrenia unresponsive to clozapine. British Journal of Psychiatry, 169 (suppl. 31), 31-40.

Green. A. I., Zalma, A., Berman, I., et al (1994) Clozapine following ECT: A two-step treatment. Journal of Clinical Psychiatry. 65, 388-390.

JAMES, D. V. \& GRAY, N. S. (1999) Elective combined electroconvulsive and clozapine therapy. International Clinical Psychopharmacology. 14, 69-72.

Thomas R. E. Barnes, Professor of Clinical Psychiatry. Department of Psychiatry. Division of Neuroscience and Psychological Medicine, Imperial College School of Medicine, Academic Centre, Ealing Hospital, St Bernard's Wing. Uxbridge Road, Ealing UB1 3EU

\title{
Early detection of antipsychotic side-effects
}

\author{
Robert Chaplin, Julie Gordon and Tom Burns
}

Aims and methods Staff from five community mental health teams (CMHTs) were trained to use structured rating scales for akathisia, tardive dyskinesia and Parkinsonism. Detection rates of these side-effects were compared for the six months before and after the intervention.
Results Fifty-seven per cent of the target professionals participated, screening 200 (52\%) elligible patients. This resulted in significant increases in the recording of all three side-effects as positive but no increase in their formal diagnosis. 